\title{
Statistical Analysis of Factors Affecting Utilization of Antenatal Care in Nepal
}

\author{
Gauri Shrestha and Ganga Shrestha \\ Central Department of Statistics, Tribhuvan University, Kirtipur, Nepal. \\ Email: gaurishrestha@yahoo.com
}

\begin{abstract}
Antenatal care provides an entry point for pregnant women to the health care system. This study investigates factors associated with the use of maternal health services as number of antenatal care visits. For analyzing the use of antenatal care health services system in Nepal, data were extracted from individual recods of a data file of NDHS 2006. The unit of analysis for this study is Ever Married Woman (EMW) who had at least one live birth in the five years preceding the survey. The sample of study consisted of 4182 EMW. The simple cross tabulation and chi square tests were used for examining the bivariate relationship and multinomial logit (MNL) model was selected for establishing linkage between number of antenatal care visits and several explanatory variables. Estimation of the parameter of this MNL models done by what is known as iteratively weighted leastsquare (IRWLS), which was identical to the algorithm of Fisher scoring and leads to maximize likelihood estimates. The results were interpreted in terms of odd ratios. The result of this study showed that women with low education level, those residing in rural areas and those with low socio-economic status were less likely to use number of ANC visits.
\end{abstract}

Key words: antenatal care; ever married woman; multinomial logit model; odd ratios; chi square.

\section{Introduction}

Maternal health refers to the health of women during pregnancy, child birth and postpartum period. Improving maternal health and reducing maternal and child mortality have been main concerns of several national summits and conferences. It begins with the international conference on Safe Motherhood held in 1987 and continued through International Conference on Population Development (ICPD) 1994 and again through ICPD +5 (Five year review of the 1994 ICPD)and then Millennium Development Goals (ICPD 1994) . World Health Organization (WHO) estimates suggested that 88 to 98 percent of all pregnancy related deaths were avoidable if all women would have access to effective reproductive health services (Kunst \& Houweling 2001). Therefore maternal health services utilization is one of the important factors to reduce the incidence of maternal death. The effectiveness of the health system in developing countries like Nepal is undermined by lack of utilization of existing facilities. Despite the government's serious commitment to deliver health facility to the door step of common people through innovative approach, the utilization of health services is still far below than any acceptable standard. Recent data in Nepal, maternal mortality ratio (MMR) is 286 per 100,000 births (NDHS 2006). Although MMR appears to have gone down over the past one decade (539 in 1996, 415 in 2000) (WHO \& UNICEF 1996, NPC 2002). The MMR is still high compared to other countries in Asia. Under such circumstances, it becomes vital to identify the factors for nonuse of maternal health services.

The indicators which are widely used to track maternal health care can be classified into four groups: Contraceptive use, antenatal care (ANC), delivery care and postnatal care (PNC). ANC is named as one of the four pilar of Safe Motherhood Initiative (WHO 1994). Antenatal care provides an entry point for women to the health care system. The objective of the system is to monitor pregnanant women regularly during their pregnancy, so that the risk factor can be identified (Mc Donagh 1996). Routine antenatal care visits may raise awareness about the need for care at delivery, care given to women or their families that enable them to seek help 
more effectively during a crisis (Palaniappan 1995). Other potential benifits of ANC are counseling on the nutrition and healthy pregnancy/ delivery behavior, HIV/AIDS and prevent low birth weight (UNPF 2006).

Coverage of ANC is define as percentage of women who receive antenatal care at least once during pregnancy for reason related to pregnancy (WHO 1996). According to Nepal Demographic Health Survey (NDHS) 2006, three in four (75\%) pregnant women receive antenatal care, about $57 \%$ of mothers who receive antenatal care reported that they were informed the sign of pregnancy complications (NDHS 2006). Quality of antenatal care is another issue. Accepted indicators of quality antenatal care include: early initiation, number of times ANC visit and medical and educational content of the visit (Philips \& Davis 1998). There has been significant improvement over the past ten years in the proportion of mothers who get antenatal care from skilled birth attendant (Doctor, nurse and midwife), increasing $24 \%$ in 1991 to $28 \%$ in 2001 and $44 \%$ in 2006 (NDHS 2006). The Nepal maternal health's program recommends at least four ANC visits: at least one visit in the first timester, at least one visit in the second timester and at least two visits in third timester. Data source shows that only $29 \%$ of mothers meet the recommended schedule (four visits). In the area of maternity care, although women

\section{Methodology \\ Data and variables}

For analyzing the use of maternal health services as number of ANC visits in Nepal, data were extracted from individual recod data file of Nepal Demographic Health Survey (NDHS) 2006. NDHS is the comprehensive survey conducted in Nepal as part of the worldwide Demographic Health Survey (DHS) project. New Era implemented it, ORC Macro provided technical support and financial support provided by the USAID. It was a nationally representative survey of 10,793 women of age group 15-49 years. The unit of analysis for this study is Ever Married Women (EMW) who had at least one live birth in the five years preceding the survey. For those EMW, who had more than one birth, only utilization behavior of maternal health services associated with most recent pregnancy within five years was considered. So the sample of this study consists of 4182 EMW.

For this study, the health behavior and services of provide tetanus immunization, malaria prophylaxis, iron and folic tablets, screening for anemia and

who receive antenatal care are relatively high (75\%) but recommended schedule is still low (29\%). Therefore the present study focuses on the number of ANC visits as maternal health services.

Utilization of health services is affected not only by access but also by demand for services which is determined largely by socio-economic and demographic factors and personal beliefs. One study on maternal health service utilization argues that characteristic of health service system is not only explanatory factors for utilization of maternal health services but other social factors and characteristic of individuals should be considered for maternal health services utilization (Matsumura \& Ghubaju 2001). In this paper, relevant literatures have been reviewed and the prospective data obtained from were analyzed to examine the factors affecting number of ANC visits. However, there are other variables that influence the maternal health services such as quality and cost of delivery services, cultural factors, prejudices, etc are also likely to influence the delivery at health facility. Due to lack of relevant data, the effect of these factors on the number of ANC visits could not be examined in this paper.

interest was the number of antenatal care visits. Therefore the number of ANC visits was choosen as dependent variable. The dependent variable was coded into three categories: no ANC visit, 1-3 ANC visits and 4 or more ANC visits. The explanatory variables were: 1) age of the respondent, 2) place of residence, 3) region, 4) religion, 5) highest level of education and 6) wealth index.

Place of residence has been included in the analysis to capture the degree of availability and accessibility to health care facilities. Religion has also been included as community factors that could facilitate or hinder health seeking behavior of a member of the community. Although others variables fairly straight forward to understand, the wealth index variable needs further explanation. It is a composite variable created from the number of variables. Information on the wealth index was based on the data collected in the household questionnaire included questions concerning the household's ownership of the number of consumer items such as television and car; dwelling characteristics such as flooring material; 
type of drinking water sources; toilet facilities and

other characteristics that are related to wealth status. Each household, for which information collected, is assigned a weight factor score generated through principal component analysis. The resulting asset scores are standardized in relation to a standard normal distribution with a mean zero and standard deviation one. These standard scores are then used to create the break points that define wealth index as poorest, poor, middle, richer and richest and again recoded into four categories: poorest, poorer, middle and rich. The description of variables used in the study is given in Table 1 .

Table 1. Description of variables.

\begin{tabular}{c|c}
\hline Variable & Description \\
\hline Number of ANC visits & $0 \rightarrow$ No ANC visit, \\
$1 \rightarrow 1-3$ ANC visits, \\
$2 \rightarrow 4$ or more ANC visits \\
Age of the respondent & It is represented by a continuous variable range from 15-49 years \\
Place of residence & $0 \rightarrow$ Rural, \\
Region & $1 \rightarrow$ Urban \\
& $0 \rightarrow$ All western, \\
& $1 \rightarrow$ Central, \\
Religion & $2 \rightarrow$ Eastern \\
& $0 \rightarrow$ Others, \\
& $1 \rightarrow$ Hindu \\
Highest level of education & 0 No education, \\
& $1 \rightarrow$ Primary education, \\
Wealth Index & Secondary and higher education \\
& $0 \rightarrow$ Poorest, \\
$1 \rightarrow$ Poorer & 2 Middle \\
& $3 \rightarrow$ rich \\
\hline
\end{tabular}

\section{Statistical Analysis}

Simple cross tabulation and chi square test were used for examining the bivariate relationship between the dependent variable and independent variables. In the process of development of the model, different types of statistical models including linear as well as nonlinear models were explored. Finally multinomial logit (MNL) model was selected for establishing linkage between number of ANC visits (Y) and several explanatory variables $(\mathrm{X})$.

In this study, the dependent variable, number of ANC visits (Y) was coded into three categories (no ANC visit, 1-3 ANC visits and 4 or more ANC visits). In MNL model, one of the categories of the outcome variable was designed as the reference category and each of the other level was compared with the reference category. In our three outcome categories, no ANC visit (category 0 ) has been designated as a reference category. We are therefore interested in modeling two main comparisons. We want to compare 1-3 ANC visits outcome (category 1) to that with no ANC visit (category 0) and we also want to compare 4 or more ANC visits (category 2) to that with no ANC visit (category 0).

There are $\mathrm{n}$ individuals in the data set, number from $\mathrm{i}=1$ to $\mathrm{n}$. If the outcome for individual $\mathrm{i}$ is in category 0 , then we let an outcome variable $\mathrm{Y}_{\mathrm{i} 0}$ be equal to 1 , otherwise $Y_{i 0}$ is equal to zero. We similarly create outcome variable $\mathrm{Y}_{\mathrm{i} 1}$ and $\mathrm{Y}_{\mathrm{i} 2}$ to indicate whether the outcome is in category 1 or category 2 .

Yij $=\{1$, if the ith incividual fall in the category $\}$

Where $\mathrm{j}=0,1,2$ and let

$$
\pi_{i j}=P\left(V_{i j}=j / X\right)
$$

denotes the probability that $\mathrm{Y}_{\mathrm{ij}}=\mathrm{j}$.

Assuming that the response categories are mutually exclusive and exhaustive then we have

$$
\sum_{j=0}^{2} \pi_{i j}=1
$$


We now consider model for probability $\pi_{i j}$, in particular we would like to consider model where their probabilities depend on a vector $X_{k}$ of covariates associated with the $i$ individual.

$\ln \frac{\pi_{i j}}{\pi_{i 0}}=\ln \frac{P\left(Y_{i j}=j / X\right)}{P\left(Y_{i b}=0 / X\right)}=\sigma_{j}+\sum_{i=1}^{g} \beta_{j k} X_{h i} \ldots \ldots \ldots \ldots$ (4)

where $\mathrm{j}=1,2$.

$\alpha_{\mathrm{j}}$ is a constant

$\beta_{j k}$ is the regression coefficient for $\mathrm{j}=1,2$ and

$X_{k},(\mathrm{k}=1,2, \ldots, \mathrm{g})$ are explanatory variables.

The MNL model may also be written in terms of probability $\pi_{i j}$ rather than the odds.

$$
\pi_{i j}=\frac{\exp \left(\alpha_{j}-\sum_{k=1}^{g} \beta_{j k} X_{k}\right)}{1+\exp \left(\alpha_{j}+\sum_{k=1}^{g} \beta_{j h} X_{k}\right)} \ldots \ldots \ldots \ldots \ldots(0 . \ldots \ldots
$$

Estimation of the parameter of this MNL model is done by what is known as iteratively re-weighted least square (IRWLS), which is identical to the algorithm of fisher scoring or Newton-Raphson, and leads to maximum likelihood estimates as shown by Mc Cullagh and Nelder(Mc Cullagh \&Nelder 1989). The computer package STATA has been used for the analysis and MNL model and $\beta_{j k}$, for $\mathrm{J}=1,2$, and $\mathrm{k}$ $=1,2, \ldots, \mathrm{g}$ are iteratively obtain with the help of this program.
The adequacy of the developed model was verified through standard statistical means such as overall goodness of fit, statistical significance of estimated parameter and multicollinearity.

For testing the significance of the beta coefficient corresponding to explanatory variables, we begin by fitting a full model (with the explanatory variables) and that to a reduced model containing only the intercepts. Then th e likelihood ratio test is calculated as

$-2 m L_{\text {reduced }}-\left(-2 m L_{\text {fwi }}\right) \times \gamma^{2}$

With degree of freedom $=$ number of parameter set to zero under $H_{0}$.

In this study $-2 \ln L_{\text {reduced }}=-4504.483$ and for the full model, $-2 \ln L f u l l=-3849.08$. The likelihood ratio chi square value is 1310.80 at 20 degree of freedom which is highly significant with $p$ value less than 0.0001 . This shows that addition of explanatory variables in the model after the intercept term is statistically significant. The coefficients of the independent variables are found to be statistically significant with $\mathrm{p}$ value less than 0.05 . Multicollinearity in the MNL model is detected by examining the standard error for the coefficients.

\section{Model Adequacy Tests}

Table 2. Bivariate analysis of number of ANC visits and explanatory variables.

\begin{tabular}{|c|c|c|c|c|c|}
\hline \multirow{2}{*}{ Variables } & \multicolumn{3}{|c|}{ Number of ANC visits } & \multirow[t]{2}{*}{ d.f } & \multirow[t]{2}{*}{ Chi square } \\
\hline & No ANC visit & 1-3 ANC visits & $\begin{array}{c}4 \text { or more ANC } \\
\text { visits }\end{array}$ & & \\
\hline \multicolumn{6}{|l|}{ Residence: } \\
\hline Urban & 14.5 & 39.0 & 46.5 & & \\
\hline Region: & & & & & \\
\hline All western & 21.3 & 48.7 & 30.0 & & \\
\hline Central & 25.8 & 44.5 & 29.7 & 4 & $37.535^{*}$ \\
\hline Eastern & 31.4 & 40.4 & 28.2 & & \\
\hline Religion: & & & & & \\
\hline Others & 31.5 & 45.5 & 23.0 & 2 & $11.819^{* *}$ \\
\hline Hindu & 27.2 & 42.9 & 29.9 & & \\
\hline Highest level education: & & & & & \\
\hline No education & 38.8 & 46.5 & 14.7 & & \\
\hline Primary edu & 19.5 & 45.2 & 35.3 & 4 & $813.810^{*}$ \\
\hline Secondary or higher edu & 6.5 & 33.8 & 59.7 & & \\
\hline Wealth index: & & & & & \\
\hline Poorest & 49.8 & 40.5 & 9.7 & & \\
\hline Poorer & 31.1 & 48.6 & 20.3 & 6 & $742.69 *$ \\
\hline Middle & 23.7 & 48.7 & 27.6 & & \\
\hline Rich & 11.1 & 39.4 & 49.5 & & \\
\hline Total & 27.8 & 43.3 & 29.0 & & \\
\hline
\end{tabular}

$(*=\mathrm{p}$ value $<0.001, * *=\mathrm{p}$ value $<0.005)$ 
The standard error larger than 2.0 indicates numerical problems of multicollinearity among the independent variables. In this study the values are less than 2.0 demonstrate the absence of multicollinearity in the developed model.

\section{Results}

Results are presented in the following two sections.

\section{Bivariate analysis}

This section will examine by means of bivariate analysis of number of ANC visits and the independent variables. Table 2 presents the bivariate analysis and chi square test.

The relationship between the number of ANC visits and highest education level of EMW shows that the number of ANC visits increases as education level increases. Women with secondary or higher education level $(60 \%)$ are four times as likely to 4 or more ANC visits as women with no education (15\%). This association is highly significant with a chi square value of 813.810 . Urban areas also have a positive and significant effect upon the number of ANC visits. It shows that the percentage of women using 4 or more ANC visits is double (47\%) than of rural women. It is commonly known that the modern health care provider tends to concentrate in urban areas which may result in the much higher utilization of 4 or more ANC visits.
The religion of the respondent and number of ANC visits statistically significant at $\mathrm{p}<0.005$. Women with Hindu religion (30\%) are more likely to have 4 or more ANC visits compared to women of other religions. Furthermore, economic variable wealth index shows stronger association with number of ANC visits. There are five fold difference in the use of 4 or more ANC visits between poorest and rich, and $50 \%$ of poorest EMW have no ANC visit.

\section{MNL model analysis}

Bivariate association between two variables does not necessarily imply a significant casual relationship between them. The magnitude and significant of these associations could change when such a simple relationship is controlled by other factors. Therefore, it is important to carry out a statistical analysis, which would incorporate more than one independent variables at a time. The most suitable analysis technique is multivariate analysis which allows the exploration of the effect of different independent variables on a dependent variable controlled for other independent variables (Tabachick \& Fidel 2007). The multiple regression analysis adopted in the present study MNL model, which would allow the identification of the effect of each of the selected independent variables on the number of ANC visits as maternal health services utilization, controlling for the effect of other independent variables. Age of household head, working status of the respondent showed no significant association with number of ANC visits when controlling for other factors. Therefore it is not included in the final model. The estimated coefficients, odd rations and standard error are presented in Table 3. 
Nepal Journal of Science and Technology 12 (2011) 268-275

Table 3. Estimation of parameters of MNL model for number of ANC visits.

\begin{tabular}{|c|c|c|c|c|}
\hline Independent variables & \multicolumn{2}{|c|}{$1-3$ ANC visits verses no ANC visit } & \multicolumn{2}{|c|}{$\begin{array}{l}4 \text { or more } \mathrm{ANC} \text { visits verses no } \mathrm{ANC} \\
\text { visit }\end{array}$} \\
\hline constant & $0.699 * *(0.299)$ & & $-0.229 * *(0.303)$ & \\
\hline Age & $0.039 * *(0.006)$ & 0.962 & $-0.068 *(0.008)$ & 0.934 \\
\hline Rural(R) & & 1.00 & & \\
\hline Urban & $0.210(0.116)$ & 1.234 & $0.531 *(0.127)$ & 1.70 \\
\hline All western(R) & & 1.00 & & \\
\hline Central & $0.510 *(0.107)$ & 1.665 & $0.296 * *(0.128)$ & 1.345 \\
\hline Eastern & $0.266^{* *}(0.101)$ & 1.305 & $0.159(0.122)$ & 1.172 \\
\hline Others(R) & & 1.00 & & \\
\hline Hindu & $0.234 * *(0.118)$ & 1.263 & $0.385^{* *}(0.149)$ & 1.470 \\
\hline No education(R) & & 1.00 & & 1.00 \\
\hline Primary & $0.370 *(0.115)$ & 1.448 & $1.409 *(0.311)$ & 2.856 \\
\hline Secondary or higher & $0.910 *(0.153)$ & 2.483 & $2.190 *(0.157$ & 8.938 \\
\hline Poorest(R) & & 1.00 & & 1.00 \\
\hline Poorer & $0.545 *(0.103)$ & 1.725 & $1.031 *(0.151)$ & 2.804 \\
\hline Middle & $0.579 *(0.115)$ & 2.136 & $1.489 *(0.156)$ & 4.432 \\
\hline rich & $1.099 *(0.120)$ & 3.003 & $2.184 *(0.152$ & 8.879 \\
\hline
\end{tabular}

The fitted model shows negative association with age while rest of the variables show positive association. Since age is a continuous variable, the presented odd ratio refers to a difference of one year in age. Respondent with one year increase is found to be .93 odds factor or $7 \%$ less of performing 4 or more ANC visits than no ANC visit. It is statistically significant at 0.01 level. Education emerged as an important variable. Respondent with primary education is found to be 1.45 odds of performing 1-3 ANC visits than no ANC visit with reference to the category of no education. Similarly respondent with secondary or higher education is found to be odds 8.94 performing 4 or more ANC visits than no ANC visit with reference category no education. Hindus are at 1.263 times odds of performing 1-3 ANC visits than no ANC visit with the reference category of other religions and 1.5 times odds of 4 or more ANC than no ANC visit compared to other religion.

The estimated coefficients of EMW under different development regions are obtained. Also some disparities exist in ANC utilization in region. The odds corresponding to central and eastern regions are 1.3 and 1.7 times more likely to 1-3 ANC visits than no ANC visit compared with all western regions. The variable is not significant for the central region to performing 4 or more ANC visits than no ANC visit. Wealth index variable is highly significant to influence on number of ANC visits. Respondent "rich" is found to have 8.9 odds of performing 4 or more ANC visits than no ANC visit compared to poorest. 


\section{Discussion}

There is no doubt that the uses of maternal health services improve reproductive outcomes (Letamo \& Majelantle 2001). It is well recognized that women's current age play important role in the utilization of maternal health services (Fosu 1994). In this study, the older women had decrease odds of ANC visits. It may be due to the improvement of education opportunities for women in recent years, younger women might have an enhanced knowledge of modern health care services. Several studies found that physical proximity of health care services; specially in the developing countries play an important role in utilization of health service (Paul 1991).This study shows that residence in rural areas use less number of ANC visits. Result of analysis reveal that among the explanatory variables, education and wealth index is the most significant determinant for increasing utilization of number of
ANC visits. Several studies have found a strong association between education and utilization of $\mathrm{MH}$ services (Becker et al 1993). It is found that mother's education is the most consistent and important determinant of use of child and maternal health services. The result from this study also supports the positive association between the education and number of ANC visits. But in rural areas, it does not seem to have greatly improved in number of ANC visits as increase in education level. Women with higher wealth index more often have resources and ability to buy health services. It is obvious from our study that as the wealth index becomes high, number of ANC visits is also increased in both urban and rural areas.This study identified the factors which affect the number of ANC visits as maternal health services utilization and it may be helpful for policy maker/service provider regarding maternal health to make future plans or program for maternal health in Nepal. 
Nepal Journal of Science and Technology 12 (2011) 268-275

\section{Acknowledgements}

It is our pleasure to acknowledge INSA-NAST bilateral exchange program for funding research at Centre for Mathematical Sciences,Kerala, India (Nov, 2010 - April, 2011). The author acknowledges gratefully the guidance and encouragement of Professor A.M. Mathai, Director of the Centre for Mathematical Sciences, Kerala, India, in this research work.

\section{References}

Becker, S., Peters, D. H., Gray,, R H., Gultiano, C and Blake R.E. 1993. The determinant of use of maternal child health services in Metro Cebu, the Philippines, Health transition review 3: 77-89.

Fosu, G.B. 1994. Childhood morbidity and health care utilization: Cross national comparison of user related factors from DHS data. Social Science and Medicine 38: 1209-1220.

ICPD.1994. International conference on population and development, program of action.(paragraph 8.22) available

at http:/www.un.org/millinniumgoals/pdf/mdg2007.pdf.

Kunst, A.E and T.Houweling. 2001. A global picture of poor-rich differences in the utilization of delivery care in safe motherhood strategies. A review of the evidence 17: ITG Press, Belgium, 297-315.

Letamo, G. and RG. Majelantle. 2001. Factors inûuencing low birth weight and pre-maturity in Botswana. Journal of BioSocial Science 33: 391-403.

Matsumura, M. and B. Ghubaju. 2001. Women status, household structure and utilization of maternal health services in Nepal. Asia Paciûc Journal 16: 23-44.

Mc Cullagh, P. and J.A Nelder. 1989. Generalized linear model, 2nd edition, Chapman \& Hall, Inc, NewYork.

Mc Donagh, M. 1996. Is ANC effective in reducing maternal morbidity and mortality?Health Policy Planning 11: 1-15.
National Planning Commission (NPC). 2002. The tenth plan. Kathmandu, Nepal.

Nepal demographic health survey. 2006. Population division, Ministry of Health and Population, Government of Nepal, Kathmandu, Nepal and Macro International, Maryland, Calverton, USA 2007.

Palaniappan, B. 1995. Roll of antenatal care in safe motherhood. Journal of the Indian Medical Association 93: $52-54$.

Paul, B.K. 1991. Health service resources as determinant of infant death of rural Bangladesh: An empirical study. Social Science and Medicine 32: 43-49.

Sanders-Philips, K., S Davis. 1998. Improving prenatal care services for low income African American-women and infants, Journal of Healthcare for Poor and Under Served 9: 14-29.

Tabachick, B.G and L.S. Fidel. 2007. Using multivariate statistics. 5th edition. Pearsons Education Inc. Boston.

United Nations Population Fund. 2006. Maternal and neonatal health in east and south East Asia. Bangkok, Thailand.

WHO and UNICEF. 1996. Estimation of maternal mortality. A new approach by WHO and UNICEF.

WHO. 1994. Mother-baby package: Implementing safe motherhood in countries: A practical guide. Document WHO/FHE/MSM/94.11. Geneva.

WHO. 1996. Coverage of prenatal care: A listing of available information. fourth edition, (WHO/FRH/MSM 196.28), Geneva. 